Gut, 1985, 26, 203-207

Methods and techniques

\title{
Plastic $\mathrm{pH}$ electrodes for the measurement of gastrointestinal $\mathrm{pH}$
}

\author{
J M RAWLINGS AND M L LUCAS \\ From the Institute of Physiology, The University, Glasgow
}

SUMmary Plastic electrodes have been developed for measuring $\mathrm{pH}$ in the human gastrointestinal tract. The electrodes have a plastic hydrogen ion sensitive membrane sealed to a length of fluid filled PVC tubing. Two recently developed hydrogen ion sensitive ligands have been examined. Their operational characteristics have been described. These electrodes have an electrical response of 52 to $58 \mathrm{mV} / \mathrm{pH}$ unit change in the range $\mathrm{pH} \mathrm{4-9,} \mathrm{with} \mathrm{a} \mathrm{diminished}$ response outwith this range. They have a low resistance value and a fast response time of one second to reach $90 \%$ of their maximum response. The electrodes can be passed down the biopsy channel of an endoscope to obtain mucosal $\mathrm{pH}$ readings under direct vision. Readings obtained in this way using plastic electrodes are comparable to those obtained with glass electrodes. Alternatively, these electrodes can be joined to a Crosby capsule, allowing continuous recording of mucosal $\mathrm{pH}$ through to the jejunum during jejunal biopsy procedures. These electrodes can be used repeatedly or may be acceptable as inexpensive disposable items for sterile clinical use.

Several techniques have been used to measure $\mathrm{pH}$ in the human intestinal tract. These include in situ measurement by radiotelemetry devices ${ }^{1}$ both shielded and unshielded free floating glass $\mathrm{pH}$ electrodes ${ }^{23}$ and measurement of aspirated luminal samples ${ }^{4}$ given that sufficient fluid is available. Aspiration of intestinal fluid is the simplest method of measuring intestinal $\mathrm{pH}$. It is unsatisfactory because only luminal samples of uncertain origin may be measured. Additionally, the technique of measuring aspirates does not allow continuous recording of intestinal $\mathrm{pH}$. Radiotelemetry devices are inconvenient because the capsule must pass through the entire intestinal tract before it may be recovered. This is a time consuming method which again only allows luminal $\mathrm{pH}$ to be measured. Glass $\mathrm{pH}$ electrodes in situ provide an adequate measuring system but the requirement for a small diameter is a severe constraint for laboratory electrode construction: commercially available glass electrodes suitable for intestinal $\mathrm{pH}$ monitoring are relatively expensive.

Address for correspondence: Dr M L Lucas, Institute of Physiology, The University, Glasgow G12 8QQ.

Received for publication 18 May 1984
Hydrogen ion selective ligands have been recently developed for use in liquid ion exchanger microelectrodes. ${ }^{56}$ These can also be incorporated in plastic membranes to provide catheter type $\mathrm{pH}$ electrodes of small outside diameter. Plastic provides a cheaper but as effective alternative to glass for the construction of suitable catheter type electrodes. We report here on the production and operating characteristics of plastic electrodes suitable for use in the human intestine. These allow $\mathrm{pH}$ readings to be obtained both from the mucosa and the lumen of the intestine if passed through the biopsy channel of an endoscope. In contrast, by attaching the electrode to a Crosby capsule, mucosal $\mathrm{pH}$ recordings may be made during routine jejunal biopsy investigations.

\section{Methods}

ELECTRODE CONSTRUCTION

Two types of plastic $\mathrm{pH}$ electrode were investigated. One consisted of a lipid soluble ligand, the octadecyloxy derivative of $\mathrm{m}$-chlorophenylhydrazonemesalonitrile $(\mathrm{OCPH})$ incorporated in a suitable

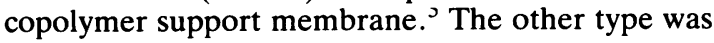


tri-n-dodecylamine (TDDA), also a hydrogen ion ligand, incorporated in polyvinylchloride. The $\mathrm{OCPH}$ based membranes were made from a solution of 1 part $\mathrm{OCPH}$ to 100 parts copolymer dissolved in dichloromethane. Similarly, TDDA based membranes were cast from a solution consisting of $30 \mu \mathrm{l}$ TDDA dissolved in $200 \mu \mathrm{l}$ of the organic solvent o-nitrophenyloctylether (o-NPOE), $1.2 \mathrm{mg}$ sodium tetraphenylborate and $100 \mathrm{mg}$ PVC dissolved in $2 \mathrm{ml}$ of tetrahydrofuran. The PVC could be either fragments of PVC tubing or PVC powder (Lonzavyl). If the PVC powder was used it was necessary to include $30 \mu \mathrm{l}$ of the plasticiser dibutyl sebacate.

To cast plastic membranes, the solvent was allowed to evaporate until a gelatinous mixture remained. Electrodes suitable for use with a Crosby capsule were cast by dipping the end of a length of PVC tubing of $1.6 \mathrm{~mm}$ outside diameter in the plastic mixture and slowly withdrawing it. A meniscus was formed over the end of the tubing which, on evaporation of the solvent, left a tough but flexible membrane. ${ }^{7}$ With OCPH based membranes evaporation of the solvent was achieved $^{8}$ by passing nitrogen gas through a specially constructed chamber suspended over crushed ice. This reduced the formation of bubbles as the membrane set. Catheter electrodes for endoscopy required a porous plug in the end of the PVC tubing for support, before the membrane was cast.

A variant of this dipping technique was found to be most suitable for electrodes used in conjunction with a Crosby capsule. These electrodes had a side mounted membrane instead of the forward facing membrane. A small hole with a diameter of approximately $1 \mathrm{~mm}$ was cut in the side of the PVC tubing at a distance of $5 \mathrm{~mm}$ from the end. Using a glass pipette it was possible to transfer a small quantity of the membrane mixture from the stock solution and smear this over the hole in the side of the tubing. This left a tough but flexible membrane sealing the hole (Fig. 1) once all the solvent had evaporated. When the hydrogen ion-sensitive membrane had dried, a solution of PVC dissolved in tetrahydrofuran was used to seal the end of the tubing.

Two types of catheter $\mathrm{pH}$ electrode were designed for measuring intestinal $\mathrm{pH}$. The simplest made entirely of plastic, consisted of two to three metres of PVC tubing with the membrane cast on one end and a chlorided silver wire, soldered to a $2 \mathrm{~mm}$ socket, inserted in the other end. The length of tubing was filled with a $\mathrm{pH} 5$ citrate buffer. The second type of electrode consisted of only a small length of PVC tubing with the remainder of the catheter electrode consisting of screened cable. Here, the length of PVC tubing was cut $10 \mathrm{~mm}$ from the membrane tip and the tubing was filled with $\mathrm{pH}$ 5 citrate buffer. A $5 \mathrm{~mm}$ length of chlorided silver wire was soldered to the core of a screened cable with a maximum overall diameter of $1.6 \mathrm{~mm}$ and a length of $2.5 \mathrm{~m}$. The silver wire was inserted into the lumen of the PVC tubing and the tubing was sealed to the cable using a water impermeable sealant. The other end of the cable was connected to a plug compatible with the voltmeter used.

\section{PRELIMINARY CLINICAL STUDIES}

Pilot studies were carried out on a series of unselected patients to see whether the electrodes functioned during routine investigative procedures. For jejunal biopsy procedures, electrodes were cemented to the tubing attached to the Crosby capsule so that the membrane of the electrode was close to the capsule aperture. The reference electrode consisted of a Pye-Unicam (340) cheek reference electrode placed in a beaker of $3 \mathrm{M}$ potassium chloride. One end of an agar bridge was

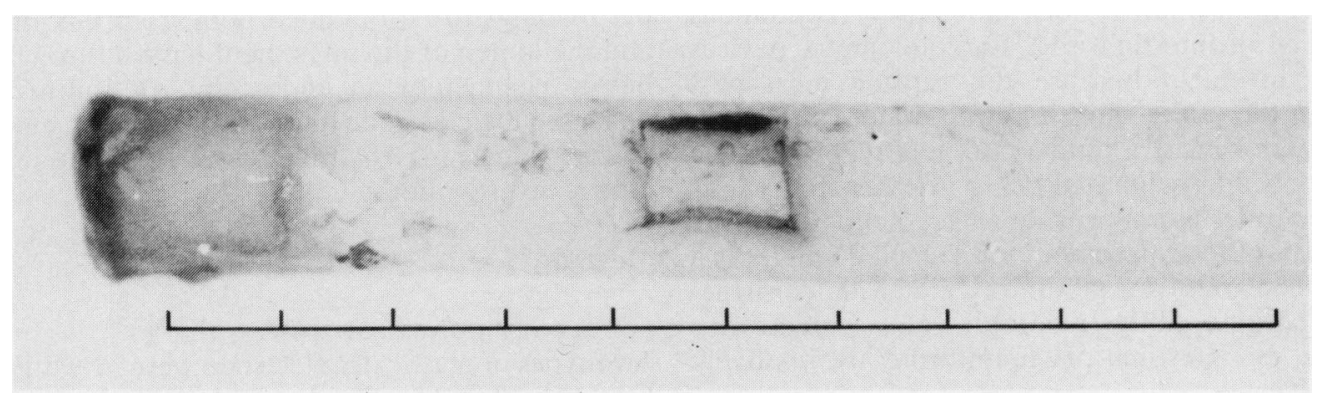

Fig. 1 Example of a plastic TDDA electrode with a side-mounted hydrogen ion sensitive membrane, the end of which is sealed with insensitive PVC membrane. Both this type and the simpler end mounted $\mathrm{pH}$ sensitive membrane types were suitable for use with a Crosby capsule. One scale division is one millimetre. 
immersed in the potassium chloride solution and the other end was positioned against the patient's buccal mucosa. This was found to be more comfortable for the patient rather than direct use of the bulky cheek electrode. The $\mathrm{pH}$ electrode and the reference electrode were connected to a portable battery operated $\mathrm{pH}$ meter (Knick Portamess 654) whose output was linked to a Watanabe (Servocorder) paper chart recorder. Readings of $\mathrm{pH}$ could therefore be obtained during the entire biopsy procedure as the capsule progressed into the jejunum.

For endoscopic investigations, catheter electrodes were introduced through the biopsy channel until the tip of the electrode was seen to protrude from the end of the endoscope. The Pye-Unicam cheek reference electrode was placed directly against the patient's buccal mucosa and both electrodes were connected to the $\mathrm{pH}$ meter. Under direct visual control, the endoscopist was able to position the electrode tip against the mucosa to obtain $\mathrm{pH}$ readings in specific areas. Both before and after use, the electrodes were calibrated in citrate and Tris buffer solutions spanning the 4-9 $\mathrm{pH}$ range. Readings obtained during the clinical investigations were corrected for any drift that had occurred.

\section{Results}

ELECTRODE CHARACTERISTICS

Both OCPH and TDDA electrodes had responses of between 52 to $58 \mathrm{mV} / \mathrm{pH}$ unit, but never less than $90 \%$ of the theoretical maximum of $58 \mathrm{mV} / \mathrm{pH}$ unit change, within the range $\mathrm{pH}$ 4-9. A diminished response (Fig. 2) was obtained outwith this range for both electrodes. Citrate, phosphate, bicarbonate and Tris-based buffers were all found to be suitable calibration solutions to use with these electrodes. Some buffers, however, were unsuitable for calibration and gave misleading results (Fig. 3). Phthalate based buffers produced an unsatisfactory response to a greater or lesser extent depending on whether an OCPH or TDDA electrode was used. For OCPH electrodes, a linear response in phthalate buffer was only obtained in the range $\mathrm{pH} 6 \cdot 5-10 \cdot 5$. The TDDA electrodes gave varying responses between the range $\mathrm{pH}$ 4-9. The $\mathrm{OCPH}$ electrodes did not respond at all when tested in commercial phthalate buffers which incorporated mercuric potassium iodide as a stabilising agent. The response shown by the TDDA electrodes in the commercial phthalate buffers was no different to that shown in phthalate buffers without the stabilising agent. This failure to respond appropriately has been attributed to the interference effect of the lipophilic organic phthalate ions ${ }^{5}$ and can result in perfectly functioning electrodes being discarded after having been tested in these buffers.

Typical electrode resistance values were of the order of 1-10 M $\Omega$ for the TDDA electrodes and 10-100 M $\Omega$ for OCPH electrodes. The low resistance meant that both types of electrode had a fast response time, reaching $90 \%$ of their maximum response in one second or less. Drift free recordings $(0.004 \mathrm{pH}$ units $/ 24 \mathrm{~h})$ were obtained when a TDDA electrode was immersed over a five day period in a pH 5 buffer solution indicating that the electrodes were comparable with glass electrodes in respect to drift. The low rate of drift that did occur can be attributed to an increase in the ionic concentration of the internal filling solution brought about by the almost imperceptible water loss through the PVC tubing. The water loss may be circumvented by immersing the entire PVC portion of the electrode
Fig. 2 Representative $p H$ responses of (a) OCPH plastic electrode (b) TDDA plastic electrode.

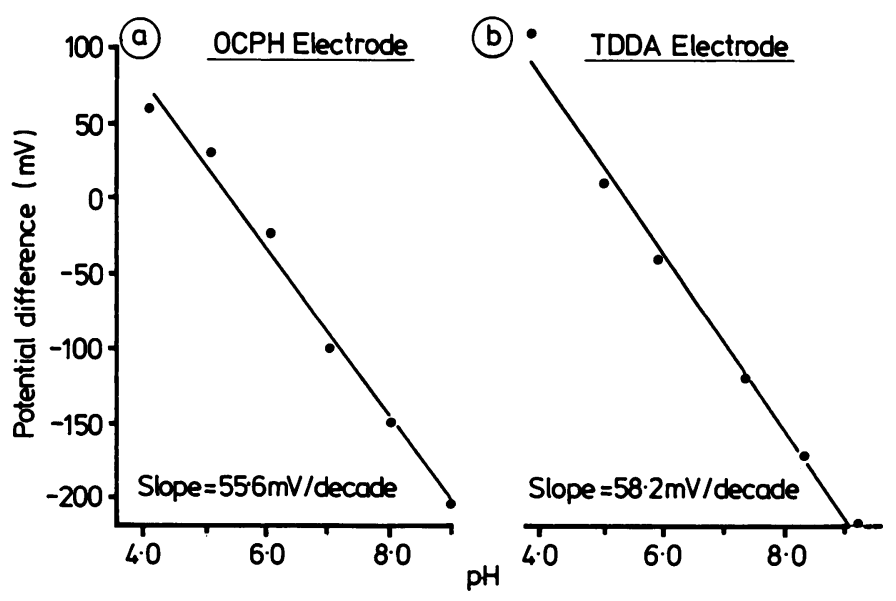


Fig. 3 Varying $p H$ responses of (a) an $O C P H$ electrode and (b) a TDDA electrode in different buffer solutions when titrated with $\mathrm{HCl}$ or $\mathrm{NaOH}$.
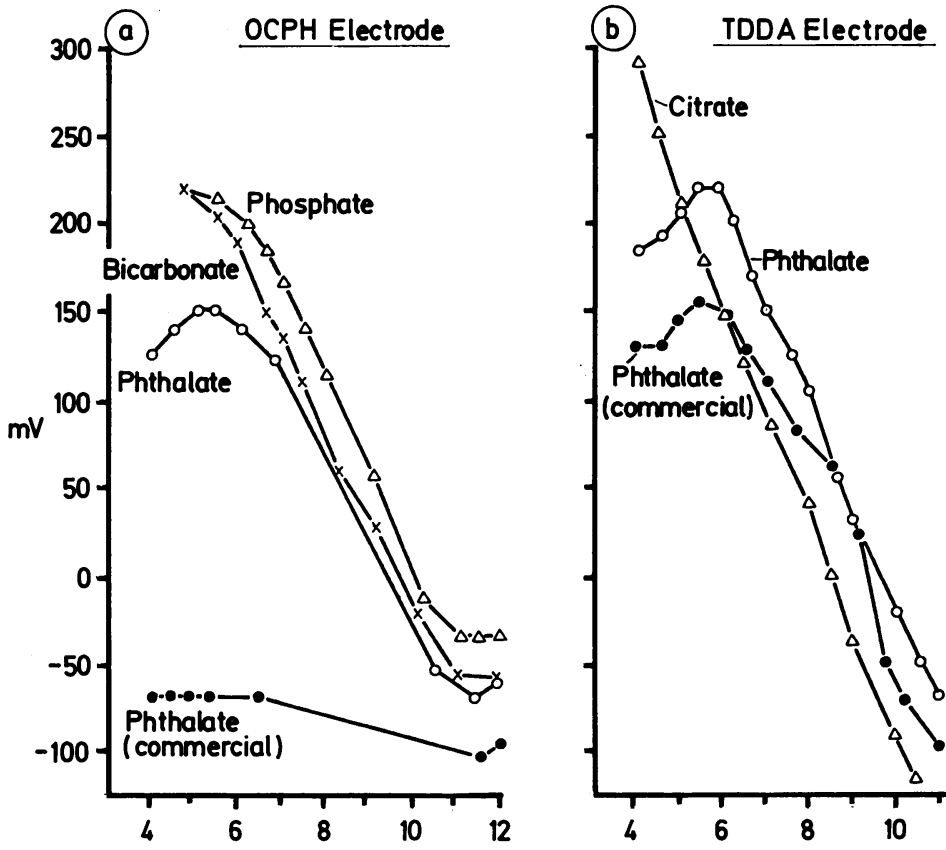

$\mathrm{pH}$ ( glass electrode) in pH 5 buffer when the electrode is not in use. With calibration before and after use, however, this is not a serious problem.

\section{PRELIMINARY CLINICAL RESULTS}

The $\mathrm{pH}$ electrode combined with the Crosby capsule provided a continuous recording (Fig. 4) of intestinal mucosal $\mathrm{pH}$ from the duodenum to the proximal jejunum, defined as the section of intestine immediately distal to the ligament of Treitz, during a typical jejunal biopsy procedure. The position of the capsule was determined by intermittent radiographic screening of the patient in the usual way. It was possible, however, to determine when the capsule had passed from the antrum, through the pylorus and into the duodenum, by the rise in $\mathrm{pH}$ from an acid value of less than $\mathrm{pH} 4$ to approximately $\mathrm{pH} 7$ when the capsule was in the duodenum.

With the aid of an endoscope, $\mathrm{pH}$ readings (Table) were obtained from the mucosa of the duodenal cap and the first part of the duodenal loop under direct visual control. The results showed that the plastic electrode gave comparable values to those obtained using a glass catheter $\mathrm{pH}$ electrode (Microelectrodes Inc, New Hampshire, USA, MI506).

Both the 'all-plastic' electrode and the 'cabletype' electrode were suitable devices for obtaining relatively noise free recordings at jejunal biopsy sessions although the recordings from the "cabletype' electrode were the most stable. Because of noise interference from movement of the endoscope and the electrical interference of associated endoscopic equipment, however, only the 'cabletype' electrode was suitable for use at endoscopy.

\section{Discussion}

We have shown that plastic electrodes are suitable for measuring intestinal $\mathrm{pH}$ and that they may be a cheap but effective alternative to glass catheter electrodes. They are somewhat more robust than glass $\mathrm{pH}$ electrodes and, if stored under the correct conditions, will function perfectly after spending

Table Specimen mucosal $\mathrm{pH}$ recordings obtained during an endoscopy procedure with either a glass or a plastic electrode introduced through the biopsy channel of an Olympus GIF-IT endoscope

\begin{tabular}{lll}
\hline Electrode region & Glass & Plastic (TDDA) \\
\hline Duodenal & $7 \cdot 00$ & $6 \cdot 88$ \\
Loop & $6 \cdot 90$ & $6 \cdot 92$ \\
Duodenal & $7 \cdot 30$ & $7 \cdot 10$ \\
Cap & $7 \cdot 30$ & $7 \cdot 18$ \\
\hline
\end{tabular}


Fig. 4 Representative trace from a TDDA electrode obtained during a jejunal biopsy procedure when the electrode was joined to a Crosby capsule. The gap in the recording was because of interruption of the monitoring procedure while the patient was screened.

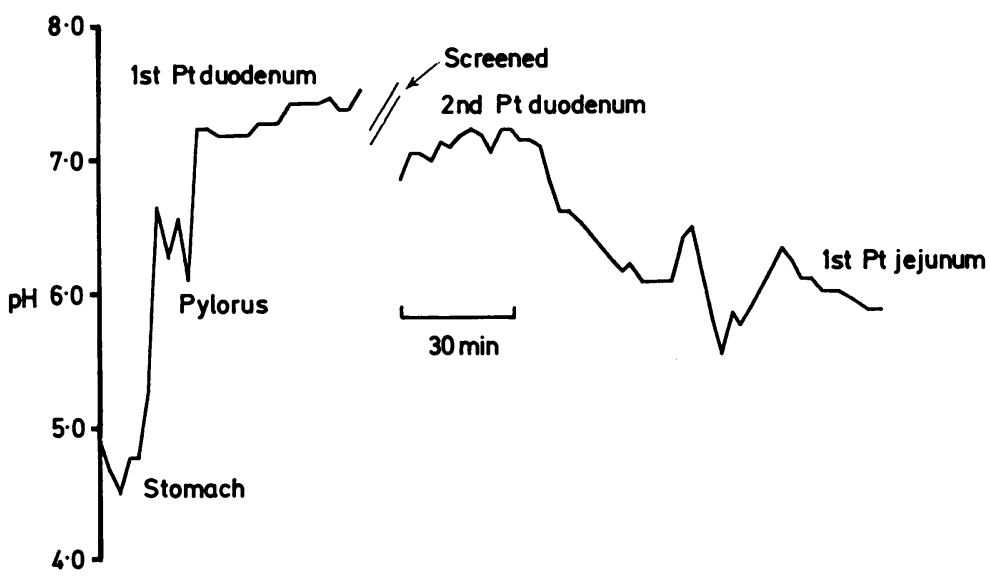

unlimited time in storage. Although they may be repeatedly used, their low cost would suggest that they could be considered as disposable items suitable for sterile clinical use. Their operative range makes them suitable for intestinal $\mathrm{pH}$ recordings but does not allow accurate measurements of gastric $\mathrm{pH}$ when this has a value of less than $\mathrm{pH} 4$. Under certain circumstances, however, such as in oesophageal reflux investigations, when only knowledge of whether a region is acid or alkaline is required, these electrodes would be suitable. Passage through the acid environment of the stomach does not appear to affect the performance of the electrode in any way.

Previous studies of intestinal $\mathrm{pH}$ using glass electrodes have concentrated on obtaining luminal $\mathrm{pH}$ values, notably by placing a plastic wire guard over the glass bulb. ${ }^{3}$ Further, it is often difficult to be certain whether a glass electrode without a guard is in contact with the mucosa of the intestine or floating free in the intestinal lumen. The two techniques described of using the plastic electrode to measure mucosal $\mathrm{pH}$ virtually ensured that the electrode was in contact with the mucosa.

The electrode, when combined with a Crosby capsule, did not impede the progress of the capsule through the intestine. It was still possible to obtain satisfactory biopsy samples from the jejunum, thus indicating that the positioning of the electrode tip close to the capsule aperture did not interfere with the functioning of the capsule. The use of these electrodes in conjunction with a Crosby capsule or an endoscope offers the possibility of conveniently obtaining additional information on ion concentrations to complement the histological and visual information provided by these procedures.

We gratefully acknowledge the generous gifts of
$\mathrm{OCPH}$ ligand from $\mathrm{Dr} \mathrm{O} \mathrm{H}$ Le Blanc, General Electric Corporation, Schenectady, NY, and of copolymer from Dr N C J Lai, Biochem International, Wisconsin. Thanks are due to the gastroenterology unit, Royal Infirmary, Glasgow for their assistance. J M Rawlings was in receipt of a grant from the British Digestive Foundation. The authors are prepared to make available to interested colleagues examples of plastic electrodes on a cost covering basis.

\section{References}

1 Meldrum SJ, Watson BW, Riddle HC. $\mathrm{pH}$ profile of gut as measured by radiotelemetry capsule. Br Med J 1972; 2: 104-6.

2 Bircher J, Mann CV, Carlson HC, Code CF, Rovelstad RA. Intraluminal and juxtamucosal duodenal $\mathrm{pH}$. Gastroenterology 1965; 48: 4.

3 Rune SJ. An electrode for $\mathrm{pH}$ measurement in the gastrointestinal tract. Scand J Gastroenterol 1968; 3: 1.

4 Benn A, Cooke WT. Intraluminal $\mathrm{pH}$ of duodenum and jejunum in fasting subjects with normal and abnormal gastric or pancreatic function. Scand J Gastroenterol 1971; 6: 313-7.

5 Le Blanc OH, Brown JF, Klebe JF, Niedrach LW, Slusarczuk MJ, Stoddard WH. Polymer membrane sensors for continuous intravascular monitoring of blood pH. J Appl Physiol 1976; 40: 4.

6 Ammann D, Lanter F, Steiner RA, Schulthess P, Shijo Y, Simon W. Neutral carrier based hydrogen ion selective microelectrodes for extra- and intracellular studies. Anal Chem 1981; 53: 2267.

7 Lucas ML, Cannon MJ. Measurement of sodium ion concentration in the unstirred layer of rat small intestine by polymer Na-sensitive electrodes. Biochim Biophys Acta 1983; 730: 41-8.

8 Cobbe SM, Poole-Wilson PA, A catheter tip pH electrode for use in man. $J$ Physiol 1979; 289: 5PA. 